\title{
Design of Psychological Counseling System Software Based on UML
}

\author{
Shan $\mathrm{Hu}$ \\ School of Education and Sports, Bohai University, Jinzhou, 121013, China \\ guoxiaotian24@163.com
}

Keywords: mental health; psychological counseling; UML; modeling mechanism

\begin{abstract}
In recent years, college students' mental health problems have become an unavoidable problem. UML-based psychological counseling system was designed in this paper in the light of defects and deficiencies of the traditional psychological counseling methods. Firstly, the UML modeling mechanism was introduced and the use case diagram in the requirement analysis phase was given; Secondly, the system of overall design was conducted. Analysizing the five functional modules of the system and its capabilities simply, and then using the E-R diagram to show the relationship between two entities; finally, specific method to evaluate and test psychological problems was given. The system not only provides professional counseling services for university students to help solve their psychological problems in a timely manner, but also can improve the efficiency of consulting.
\end{abstract}

\section{Introduction}

As society progresses, psychological problems has been more attention to the majority of people currently. Students are special groups that they are maturing but not fully mature, and their psychological quality is fragile and easy to produce a variety of psychological problems [1]. The mishandling of these pressures and lack of timely counseling will lead to an increasing number of mental health problems. Therefore, mastering students' psychological problems, carrying out psychological counseling in a timely manner and correcting students' unhealthy psychology and personality can improve the psychological quality of students, and tap college students' mental potential. Related researches show that psychological counseling is basically a blank in many of our universities, and this work has a special significance with the growing enrollment [2]. However, there are many limitations in traditional consulting methods, such as single counseling model, long consultation period, difficulties in organizing and saving files and poor security. The above shortcomings restrict the smooth development of college mental health work to a certain extent. Therefore, it is imperative to strengthen propaganda for psychological counseling and build a well-functioning psychological counseling system for students and teachers to communicate timely. Network psychological counseling system can keep secret for students and build an equal and easy counseling relationship, and it has advantages of freedom choice, rich information, convenient, easy to think and analysis, easy to store and query cases. It has many advantages that traditional way of psychological counseling can't replace [3].

\section{Requirement Analysis}

Requirement analysis is an important task of the software life cycle, and it is also a crucial work. Only through requirement analysis, can the overall concept of software functionality and performance, described as specific software requirements specification, thereby laying the foundation for software development. Requirement analysis is important, because with decision-making, direction, strategic role, plays a decisive role in the software development process. Whether it is in the process of learning software engineering or in software development practice, be sure to have enough attention to requirement analysis, which is to develop the right, an important guarantee for high-quality software. The importance of the entire software development process mainly in the following points: (1) The requirement analysis is an effective way to get the user needs. In-depth understanding of software requirements, is the prerequisite for the success of the 
software development work, regardless of the software engineers to design and coding work done to how well, can not really meet the user requirements of the software, users will only be disappointed. (2) The requirement analysis is the key factor in determining project success. Requirement analysis is the beginning of the project, is the cornerstone of the project construction, construction in the past failed projects, $80 \%$ were due to uncertainty caused by the requirement analysis. So one of the key factors in the success of the project is to grasp the extent of the requirement analysis. (3) The requirement analysis is the bridge of systems analysis and software design. Requirement analysis process is to determine the process of the users requirements, users know their own requirements, but don't know how to use computer technology to achieve; and software designers and programmers, often lack the skills to understand practical business operation process and business process. So by specially trained systems analysts, it fills the gap between business areas and the computer world. (4) The requirements analysis is an important stage in the control software quality. At each stage of the software life cycle, using scientific management methods and advanced technological means, and before the end of each stage, are strictly examined from a technical and management perspectives, after qualifying began the next stage of work.

Over the past decade year, more and more people recognize that requirement analysis is the most critical part in the process of software project. If the analysts are unable to correctly recognize the needs of customers during requirement analysis, then the final software will be impossible to achieve the customer's needs virtually, or software project cannot be completed within the stipulated time [4]. There are a lot of methods for requirement analysis, such as process-oriented approach, object-oriented approach, prototyping approach and structured approach, this paper introduces the case modeling method. UML is the unified modeling language, which is independent of the development process. UML is a standard modeling language to design software blueprint, and it is a visual object-oriented model language. From the perspective of system engineering, UML is an analysis tool of software structure. Maximum use of this modeling language is to use a graphical to describe the various objects in the real world, so that all system designers can make the whole development process completely standardized when building the system from the system process analysis, system requirement, and object model definition to object design. Use case model describes system functions understood by the external actors. It includes the following elements: (1) Actor: actor is present in defined system external and human or other systems that can interact with the system; (2) use case: use cases is used to represent services provided by the system; (3) communication association: communication association represents the corresponding relationships between actors and use cases, it indicates which services are used by actors. Use case model of psychological counseling system shows in Fig. 1.

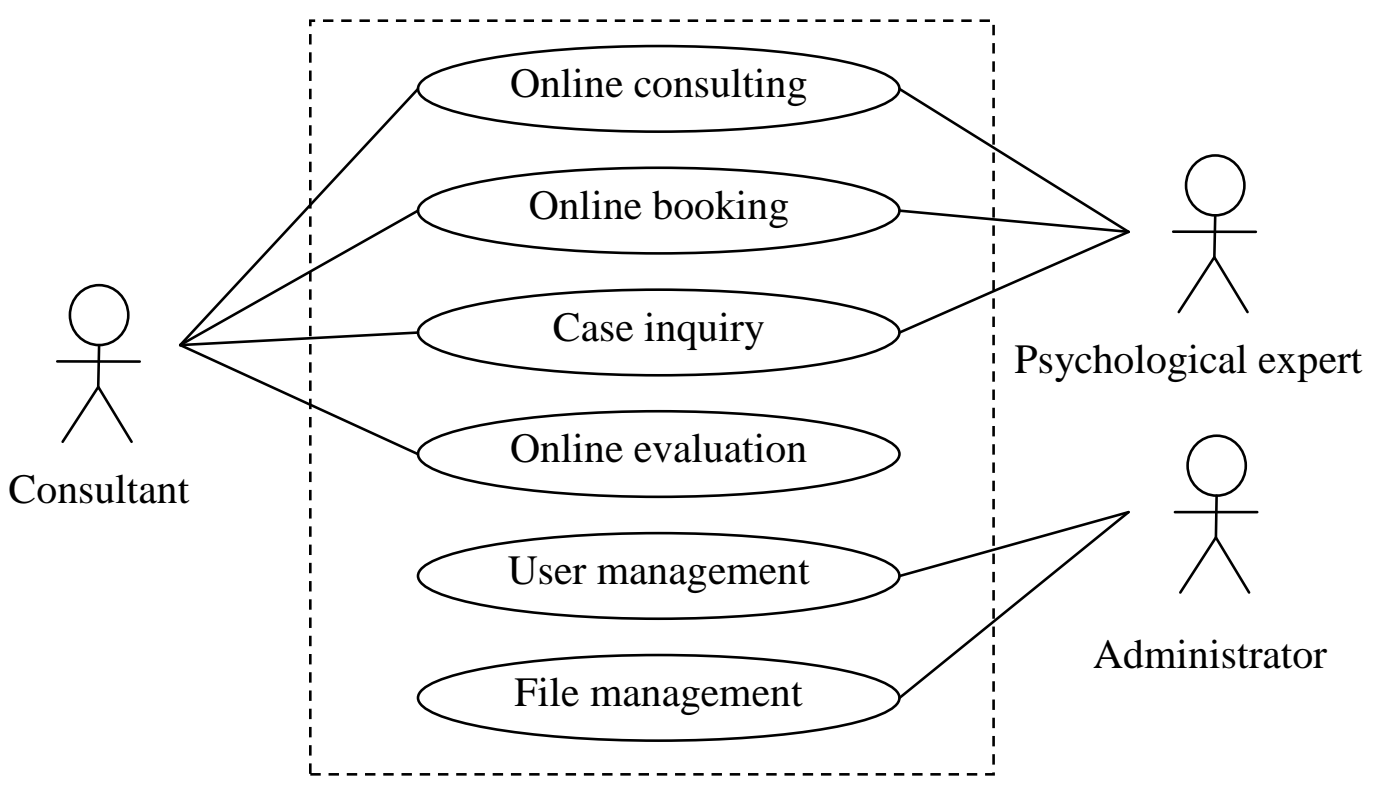

Fig. 1. Use case model of psychological counseling system 


\section{System Design}

System design is the next step of requirement analysis. The main tasks are: to achieve the functionality and performance of the software requirements model, consider the software implementation environment, through the system analysis model comprehensive analysis and refinement, to determine the design modeling of software system. Software development process after the requirements analysis phase, have made it clear the user's requirement, which is already solved the issue of software to be developed "what to do", and these identified requirements should have detailed description and full expression in the software requirements specification. After entering the software design phase, can begin to examine the implementation work of software requirements, that is to begin to solve the problem of "how to do" [5].

The work of each step of software design phase are described as follows: according to data flow diagram of the outline design, determine the relationship between the major components of the software system; detailed design based on control specifications, state transition diagrams and process specifications, will form a software architecture part converted into the process description of an integral part of software; interface design according to the data flow diagram, to define interaction between the various components within the software, software and other collaborative system, and the user and the software; database design data of data objects description, data objects and relationship of E-R diagram described, and detailed data content of the data dictionary description converted into the data structures of implement the software needs.

The purpose of the online counseling system is to build an interactive platform for psychological counseling on the network, and taking advantages of the network to conduct interactive information platform of mental health education, counseling and psychological testing service and others related to mental health [6]. According to the objectives of system and functional requirements, psychological counseling system can be divided into five functional modules, and they are user management, online evaluation, case inquiry, online booking, and online consulting.

(1) User management module: this module includes four sub-modules, respectively user registration, user profile modification, user deletion, and user login. It mainly completes that administrators can maintain, modify, and delete the users' information in the system;

(2) online evaluation module: in online evaluation module, students are able to complete the self-psychological tests to help students find their own problems as early as possible, so that they can find solutions to solve problems;

(3) case inquiry module: case inquiry module can achieve inquiry function for students to query typical cases. Students can carry out self-psychological treatment effectively by querying and learning related typical cases to solve their own psychological problems timely;

(4) Online booking module: the main function of this module is to appoint time. In order to improve efficiency and reduce the situation of queuing crowded, students should book time with experts and then communicate with experts at the appointment time to solve their problems;

(5) online consulting module: the main task of consulting module is to achieve psychological counseling. Students and experts exchange and solve psychological problems timely on the network.

The users' requirements got in requirement analysis can be abstracted to information structure that process of conceptual model is the conceptual structure design. Conceptual structure is an abstraction of the real world, which artificial processing actual people, objects, things and concepts to extract the common characteristics of people concerned about, and ignoring nonessential details, and using a variety of concepts to describe these characteristics accurately [7]. Currently, the main tool is the E-R diagram to describe conceptual model. E-R data model provides entities, attributes and relationships to represent relationships between entities, thus simulating the real world. There are two many-to-many relationships in the system, a consultant can consult a number of psychological experts, but a psychological expert can provide advices for more consultants; an administrator can manage information of many psychological experts, and a psychological expert can also be managed by multiple administrators. According to the above analysis, E-R diagram of psychological counseling system shows in Fig. 2. 


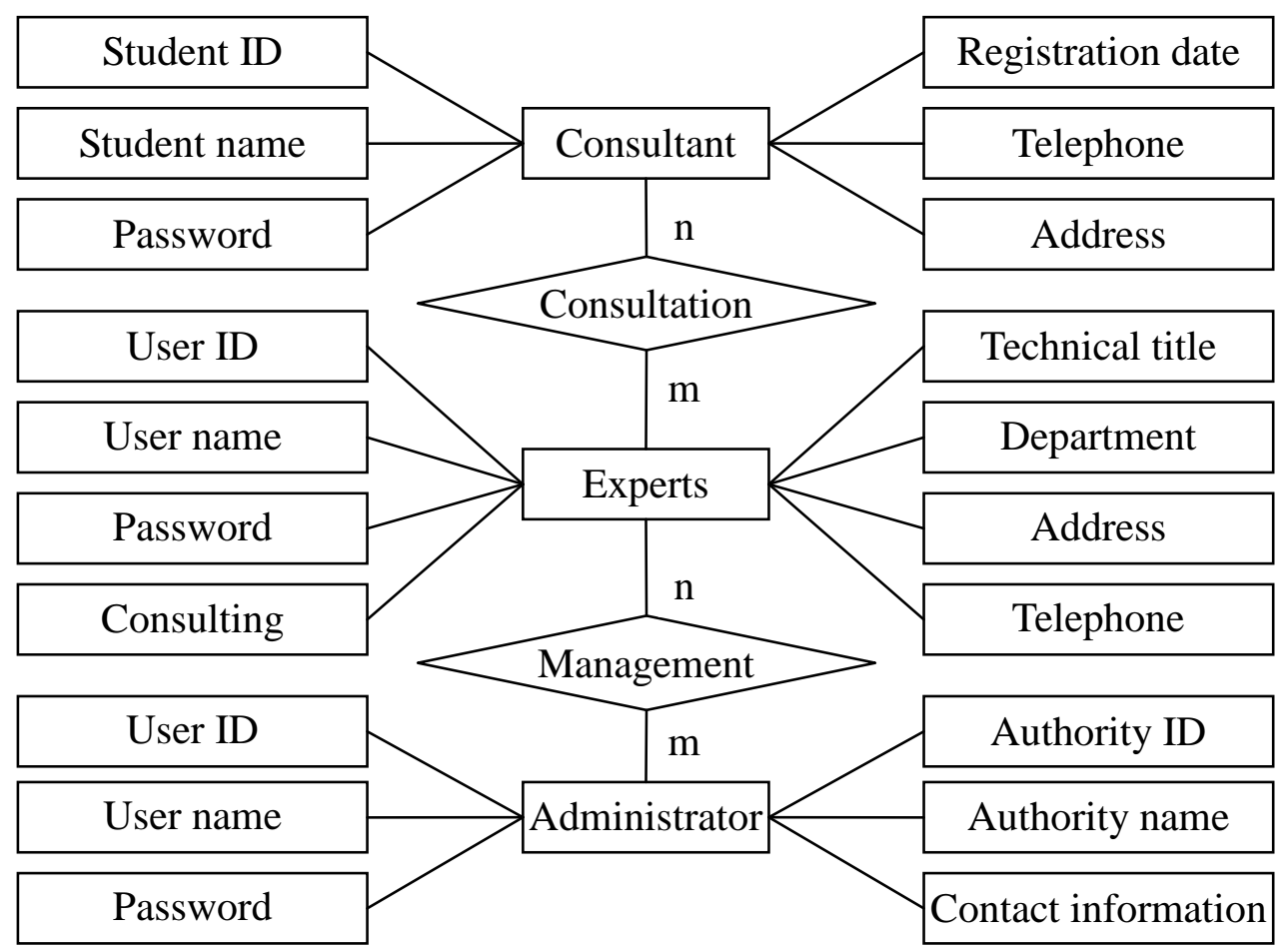

Fig. 2. E-R diagram of psychological counseling system

\section{Psychological Testing and Assessment}

Psychological test is an important tool to study and evaluate mental health, using standardized scale tool that has been certified to stimulate testers and then recording the reaction results of the test and quantifying results. Finally, the results are analyzed and assessed by analyzing the trail of behaviors [8]. Combined with computer system testing testers' psychology, the automation of forming analysis report process and analyzing testing process, scoring process and testing results can be achieved. There are many common psychological test scales, such as EPQ, UPI and 16PF. Due to a larger number of test scales, so we can use the current international SCL-90 with good reliability and validity to illustrate the norm of national college students. The scale has 90 projects, which includes contents of psychotic symptomatology, from feeling, emotion, thought, consciousness, behavior to habit, relationship, sleep and eating and so on are involved. We can use 10 factors to reflect psychological symptoms in 10 aspects. SCL-90 provides the following analysis indicators:

(1) The total scores $(S)$ : The sum of 90 projects $\left(\mathrm{S}=\sum_{\mathrm{i}=1}^{90} \mathrm{x}_{\mathrm{i}}\right)$, it can reflect the severity of their condition.

(2) The total average $(A):(\mathrm{A}=\mathrm{S} / 90)$, it shows that the feel of subjects in which degree of 1-5.

(3) The number of positive items $\left(m_{+}\right)$: the number of items that individual points $\geq 2$, and it represents how many items are "symptoms" for subjects.

(4) The number of negative items ( $\left.m_{-}\right)$: the number of items that individual points $=1$, and it represents how many items are "no symptoms" for subjects.

(5) The average scores of positive symptom $\left(M_{+}\right):\left(\mathrm{M}_{+}=\left(\mathrm{S}-\mathrm{S}_{-}\right) / \mathrm{m}_{+}\right)$, it reflects the bad projects of subjects, and the severity in which ranges.

(6) The factor scores (T): factor scores= the project scores of a factor/the number of items of a factor $\left(\mathrm{T}=\mathrm{S}_{\mathrm{T}} / \mathrm{M}_{\mathrm{T}}\right)$.

The norm of national youth shows in Table 1.

\section{Conclusion}

Mental health problem of college students is one of the focal problems, various colleges and universities see students' mental health problems as importance [9]. Establish psychological 
counseling system on the network, the network has the features of confidentiality, real-time, convenience and concealment. And these features provide convenience for psychological counseling, and create an unlimited development platform. Computers play the full role in psychological counseling, computers can preserve the counseling contents, and the obtained data should be analyzed to get laws. School counseling center can refer the valid data, thus better to prevent and solve psychological problems in college students. Practice has proved that the online counseling system is feasible, and it has the advantages of low cost, easy-to-access and real-time interaction and so on. It achieved one-on-one online interaction consulting service regardless of time and space restrictions to meet the variety of counseling needs for majority of psychological counselors. Implementation of the system has great significance in improving the psychological quality of students and improving the efficiency of psychological experts to provide support for digitization and high efficiency of school management, which has a certain value [10].

Table 1. The norm of national youth in SCL-90

\begin{tabular}{|c|c|c|c|c|c|c|c|c|c|}
\hline Index & $\begin{array}{c}\text { Somati- } \\
\text { zation }\end{array}$ & Force & $\begin{array}{c}\text { Sensit- } \\
\text { ivity }\end{array}$ & $\begin{array}{c}\text { Depres- } \\
\text { sion }\end{array}$ & Anxiety & Hostile & Terror & $\begin{array}{c}\text { Parano- } \\
\text { id }\end{array}$ & $\begin{array}{c}\text { Traum- } \\
\text { as }\end{array}$ \\
\hline \multirow{2}{*}{ Norm } & $1.34 \pm$ & $1.69 \pm$ & $1.76 \pm$ & $1.57 \pm$ & $1.42 \pm$ & $1.50 \pm$ & $1.33 \pm$ & $1.52 \pm$ & $1.36 \pm$ \\
& 0.45 & 0.61 & 0.67 & 0.61 & 0.43 & 0.57 & 0.45 & 0.60 & 0.47 \\
\hline
\end{tabular}

\section{Acknowledgement}

This work is supported by "12th Five Year Plan" of educational science planning project of Liaoning province: Special subject research base for 2015 (JG15ZXY04). Subject name: Current situation investigation and countermeasure research of college students' mental health education for Liaoning province. 


\section{References}

[1] H. M. Gu, "Research and Design of College Counseling System Based on Java NIO," Fujian Computer, vol. 28, no. 11, pp. 116-118, 2012.

[2] Y. M. Ou, "Design of Psychological Consultation System in University Based on Web," Science Mosaic, vol. 23, no. 11, pp. 39-43, 2010.

[3] G. Y. Chen, "Design and Implementation of College Counseling Network System Based on jQuery," Journal of Wuhan Institute of Technology, vol. 33, no. 10, pp. 103-106, 2011.

[4] Baidu Encyclopedia, "Requirement Analysis," http://baike.baidu.com/view/43.htm, 2014-9-20.

[5] Baidu Encyclopedia, "Software design," http://baike.baidu.com/link?url=1mICLQH, 2015-7-21.

[6] X. Q. Wang, "Design and Implementation of University's Internet Psychological Counseling System," Modern Computer, vol. 27, no. 11, pp. 109-111, 2010.

[7] J. Zhang, "Development and Design of College Psychological Counseling System," Doctor's degree of University of Electronic Science and Technology of China, 2011.

[8] Q. H. Zeng, "The Design and Implementation of University Psychological Consultation Website," Doctor's degree of Jilin University, 2012.

[9] J. Liu, "Students' Mental Health Advisory System," Doctor's degree of Shandong University, 2013.

[10] X. Y. Li, "The Construction of New University Counseling System," Public Communication of Science \& Technology, vol. 2, no. 23, pp. 96, 2010. 